\title{
3 Research Suare \\ Perivascular Epithelioid Cell Tumor (PEComa) of the Liver: a Report of Two Cases
}

\section{Yi-xiang Li}

The First Hospital of Lanzhou University

\section{Wei-long Lv}

The First Hospital of Lanzhou University

\section{Li-li Wang}

The First Hospital of Lanzhou University

\section{Meng-meng Qu}

The First Hospital of Lanzhou University

\section{Yuan-hui Zhu}

The First Hospital of Lanzhou University

Jun-qiang Lei ( $\square$ Leijq2011@126.com)

The First Hospital of Lanzhou University

\section{Case Report}

Keywords: Liver, Neoplasm, Tomography, X-ray computed, Magnetic resonance imaging

Posted Date: February 21st, 2022

DOI: https://doi.org/10.21203/rs.3.rs-1374676/v1

License: (c) (i) This work is licensed under a Creative Commons Attribution 4.0 International License. Read Full License 


\section{Abstract}

Background: Perivascular epithelioid cell tumor (PEComa) is an uncommon tumor of mesenchymal origin which can arise at a variety of visceral and soft tissue sites. It most often occurs in the uterus, but develops in the liver very infrequently, only $8 \%$ of PEComa cases.Here, we present two pathologically proven cases of PEComa of the liver, retrospectively discuss the clinical, imaging and histological features.

Case presentation: Case1:A 54-year-old female patient with intermittent epigastric discomfort for 2 years and aggravated for 5 days.The laboratory examinations were normal.Computed tomography (CT) showed the lesions were located on the right lobe The discomfort had resolved after surgery and PEComa was diagnosed after pathological examination of the surgical specimen. The patient has been followed-up for 5 years without recurrence.Case2:A 30-year-old woman was found to have a mass of liver incidentally during regular physical examination after breast cancer surgery. On contrast enhancement CT, a well demarcated mass was found. After operation, pathological diagnosis was established as malignant PEComa of the right liver. Neither primary recurrence nor metastasis was found during the 2year follow-up.

Conclusions: The imaging manifestations of liver PEComa have certain characteristics. When the tumor density is not uniform or contains fat, and thickening vascular shadow appears around the lesion, combined with clinical and laboratory examination, suggestive diagnosis can be made.

\section{Background}

PEComa is a rare stromal tissue-derived tumor ${ }^{[1]}$ which show perivascular epithelioid cell differentiation, and the incidence is rising. The World Health Organization defines PEComa as a family of related mesenchymal neoplasms which share a distinctive cell type, the perivascular epithelioid cell or "PEC' (which was morphologically described first in 1944 by Apitz and it was designated as an "abnormal myoblast" in renal angiomyolipoma, but there is no known normal tissue counterpart). In 1996, Zamboni ${ }^{[2]}$ put forward the concept of PEComa for the first time, and four years later, the case report of PEComa of the liver was first reported by Yamasaki ${ }^{[3]}$.The "family"now includes angiomyolipoma, lymphangiomyomatosis, clear cell "sugar" tumor of the lung, and a group of rare, morphologically and immunophenotypic lesions that are simply termed PEComa ${ }^{[4]}$.This tumor always composed of nests and sheets of usually epithelioid but occasionally spindled cells with clear to granular eosinophilic cytoplasm and a focal connection with blood vessel walls.PEComa is predominant in the uterus, it also occurs in a wide variety of organs, such as the kidneys, bladder, prostate, lungs, pancreas, and liver ${ }^{[5]}$. Compared to other liver tumors, these lesions are uncommon and difficult to identify. But due to the development of diagnostic procedures and there are characteristic immunohistochemicall indicators to confirm the diagnosis, we now diagnose PEComa more often. Our cases add to the volume of primary hepatic PEComas, and contribute to increase awareness and understanding of this rare tumor. 


\section{Case Presentation}

Case 1: A 54-year-old female patient with intermittent epigastric discomfort for 2 years and aggravated for 5 days. The patient had a nonsignificant past medical history, no history of recent illness and/or trauma and was not receiving any medication at the time of referral.The laboratory examinations were normal.Computed tomography (CT) showed that the lesions which one were located on the right lobe was heterogeneous in density and were heterogeneously enhanced in arterial phase images.No portal vein tumor thrombus and retroperitoneal enlarged lymph nodes were found, and there was no obvious invasion of the surrounding organs. Immunohistochemistry: ki67 (<5\%), CD34 (vascular rich), Vimentin $(1+)$, HMB $(2+)$.Pathological diagnosis: perivascular epithelioid cell tumor (PEComa). The patient has been followed-up for 5 years without recurrence.

Case 2: A 30-year-old woman was found to have a mass of liver incidentally during regular physical examination after breast cancer surgery.Blood, stool and urine routines were normal. On contrast enhancement CT, a well demarcated mass, sized $2.4 \mathrm{~cm} \times 3.1 \mathrm{~cm}$, was found with significant and uniform enhancement in the Arterial phase. There was no evidence of fatty density, calcification and necrosis in the mass. Liver metastasis of breast cancer was considered before operation. Immunohistochemistry: CD31 (vascular +), CD34 (vascular +), SMA (partial +), Smur100 (adipocyte +), ki67 (> 5\%), Vimentin (1 +), HMB (1+), CD117 (focus +), Melan-A (1 +).After operation, pathological diagnosis was established as malignant PEComa of the right liver. Neither primary recurrence nor metastasis was found during the 2-year follow-up.

\section{Discussion And Conclusions}

PEComas are rare mesenchymal tumors with unpredictable behavior,the etiology remains uncertain. They are characterized by epithelioid cells, which stain with melanocytic markers, associated with spindle cells reactive for smooth muscle markers, nearly all PEComas show immunoreactivity for both melanocytic (HMB-45 and/or melan-A) and smooth muscle (actin and/or desmin) markers ${ }^{[6]}$.More specifically, these cells are immune to melanocytic markers, have an epithelioid appearance and a transparent eosinophilic cytoplasm, and show a perivascular distribution. This heterogeneity makes it difficult to diagnose by imaging, biopsy and other techniques before operation. Because of low morbidity, there is a lack of comprehensive understanding of this hepatic lesion.

PEComas show a marked female predominance, with a male-to-female ratio of $6: 1 .{ }^{[7,8]}$. Usually, the focus is single.In the past, it was considered to be a benign disease,generally exhibits an inert biological behavior.However, multifocality is also reported more frequently in cases involving malignant lesions.Since 2000, some studies have shown that PEComas can display characteristics of both benign and malignant tumors. What's more, PEComas have a wide variety of presentations and behavior,recurrences or metastases can be observed in patients with tumors exceeding $5-7 \mathrm{~cm}$ in size in some studies ${ }^{[9,10]}$. Malignant PEComas originating from the liver can affect many abdominal organs simultaneously, including the omentum, resulting in massive bleeding into the peritoneal cavity. 
According to some histologic features such as growth pattern,size of the lesion, mitotic activity, necrosis,nuclear grade, and vascular invasion, PEComas have been divided into 3 prognostic categoriesbenign, uncertain malignancy potential, and malignant.But Criteria to define the biological behavior of these rare lesions have not been clearly defined. Currently, the most useful features to predict poor outcome are tumor size $>5 \mathrm{~cm}$, high nuclear grades, infiltrative growth patterns and cellularity, mitotic rate $>1 / 50$ high-power fields (HPF), necrosis, and vascular invasion, as proposed by Folpe in $2005^{[11]}$. Therefore, clear diagnosis and early intervention is extremely important for the prognosis of patients, but diagnose them preoperative is difficult.First of all, PEComas are commonly asymptomatic, the symptoms(such as abdominal pain,increased tension of the abdominal wall, constipation, or signs of ileus) are always caused by other diseases or occur in cases involving very large PEComa lesions.Secondly,the disease is not significantly associated with hepatitis and liver cirrhosis. Laboratory examinations in patients with PEComa don't reveal any specific abnormalities,Serum AFP, carcinoembryonic antigen, and Ca19-9 concentrations are also within the normal ranges. The most important thing is that PEComas mimic features of other hepatic neoplasms. At present, there are no characteristic imaging findings.Tumors typically had well-defined borders and show uniform or uneven enhancement on arterial phases.Some of the lesions contain fat and malformed blood vessels. These imaging features are non-specific, similar to many other liver tumors, including hepatocellular carcinoma (HCC) and focal nodular hyperplasia (FNH) among others, but the unique feature of PEComas is the coexpression of at least one smooth muscle and melanocytic markers. That is to say,these are identified immunohistochemically by the expression of Human Melanin Black-45 (HMB-45), Melan-A and Smooth Muscle Antigen (SMA) which are seen in the majority of tumors. Among them,HMB45 is the most sensitive indicator, because there is no expression in primary liver tumors except hepatoblastoma in children, but it is positive in almost all PEComas ${ }^{[12]}$.

If we focus on the imaging performances,PEComas with high arterial vascularization as a consequence of rich vascularization from the branches of the hepatic artery have been described. In diagnostic studies, the main features of these tumors are the strong enhancement in the arterial phase.The presence of adipose tissue, which is easier to detect, is typical only for some PEComas,such as case-one.So when PEComa is blood-rich and contains adipose tissue, it should be differentiated from liposarcoma, steatosis of hepatocellular carcinoma and liver adenoma:(1) Primary liposarcoma of the liver is rare but more common in male patients, its imaging findings are difficult to distinguish from PEComa of the liver, but the fibrous septum within the tumor and a small amount of local fat components are helpful for the definite diagnosis. (2) The content of steatosis in HCC is very few, and the patient always has a history of hepatitis and liver cirrhosis.AFP increases generally, and it is easy to be complicated with necrosis, portal vein tumor thrombus, enlarged peripheral lymph nodes, which can be distinguished by clinical diagnosis. (3) Hepatic adenomas contain true capsule and are prone to fatty necrosis, but they often occur in special populations and are common in young women with a long history of oral contraceptives. When there is no fatty density inside the mass, it is difficult to differentiate from many kinds of liver diseases, so it is necessary to think more according to clinical and laboratory information : (1) when PEComa did not show contrast "wash-out", which overlap with features of benign, well vascularized tumors such as focal 
nodular hyperplasias (FNH) and hemangiomas. These, however, should not be mistaken, as FNHs show very homogenous enhancement and in turn, hemangiomas have a typical blood pooling appearance, which both differ significantly from heterogenous enhancement of PEComas. (2) when the enhanced lesion showed "fast in and out", it is often misdiagnosed as primary hepatocellular carcinoma which one's some laboratory and clinical characteristics have been described before. However, the AFP value of PEComa of liver is generally not high, and the expression of HMB-45 is positive. But HCC is generally supplied by small hepatic artery, and the proportion of blood supply artery in the focus is relatively low, while PEComa is rich in twisted abnormally dilated thick-walled vessels, and strips and punctate vessels can be seen in the mass. This sign is of high value in differentiating the two neoplasms.

Due to the rarity and atypical symptoms of primary hepatic PEComa, it is easy to delay the timing of treatment,thus we presented two pathologically proven cases of PEComa of the liver and inspected the importance of thoughtful examination in the diagnosis of this lesion, and the necessity of a more sensible approach and broad investigation for the stratification of the biologic behavior of PEComas. In our study, The patients are females with no background of liver cirrhosis, the focuses were located in the right liver and had no typical clinical symptoms, which are in accordance with the epidemiological report.Above all, the natural history of primary hepatic PEComas is quite varied and not yet well established or predictable. Presentation ranged from a palpable abdominal mass to acute abdomen. Usual treatment is surgery for benign tumors and chemotherapy including mTOR inhibitors for malignant tumors.In short, for liver space-occupying lesions with no history of substantial liver disease, thickened and twisted blood vessels and negative tumor markers, the possibility of PEComa should be considered in addition to common diseases, but the identification of benign and malignant tumors should also be combined with pathological and immunohistochemical results.In view of the fact that the biological behavior of liver PEComa is not entirely known at present, long-term follow-up of postoperative patients is a necessary clinical management measure. Hopefully, further research will allow accurate prediction of the behavior of this lesion and establish firm criteria for discrimination between malignant and benign tumors. At the same time, further research into the etiology of PEComa may yield new drug targets for treating this distinctive tumor.

\section{Declarations}

\section{Acknowledgements}

We are grateful for surgery support Jun Wang and the personnel of the nursing department. This work was supported by grants from the The First Hospital of Lanzhou University, (Grant No. Ldyyyn2020-14)

\section{Authors' contributions}

Yi-xiang Li ,Wei-long Lv,Li-li Wang,Meng-meng Qu and Yuan-hui Zhu collected the data. Yi-xiang Li ,Weilong Lv and Jun-qiang Lei did the analysis and interpretation of data; Yi-xiang Li and Wei-long Lv conceptualized and wrote the manuscript. Grant to Clinical Foundation Of The First Hospital of Lanzhou University financed this study. All the authors approved the version to be published. 


\section{Funding}

Funding from the Clinical Foundation Of The First Hospital of Lanzhou University, (No. Ldyyyn2020-14) is gratefully acknowledged.

\section{Availability of data and materials}

Data are available on request from the authors.

\section{Ethics approval and consent to participate}

This case was approved by the institutional ethical review board of The First Hospital of Lanzhou University of Science and Technology. Written informed consent for the present study was obtained from the patient.

\section{Consent for publication}

The manuscript has not been and will not be a podium or poster meeting presentation.

\section{Competing interests}

No potential conflict of interest relevant to this article was reported.

\section{References}

1. Folpe AL, Kwiatkowski DJ. Perivascular epithelioid cell neoplasms: pathology and pathogenesis. Hum Pathol. 2010 Jan;41(1):1-15. doi: 10.1016/j.humpath.2009.05.011. Epub 2009 Jul 15. PMID: 19604538.

2. Fletcher, C.D.M.; Bridge, J.A.; Hogendoorn, P .C.W.; Mertens, F.World Health Organization Classification of Tumours of Soft Tissue and Bone, 4th ed.; IARC Press: Lyon, France, 2013; pp. 230231.

3. Zamboni G, Pea M, Martignoni G. Clear cell "sugar" tumor of the pancreas. A novel member of the family of lesions characterized by the presence of perivascular epithelioid cells. Am J Surg Pathol. 1996 Jun;20(6):722-30. doi: 10.1097/00000478-199606000-00010. PMID: 8651352.

4. Yamasaki S, Tanaka S, Fujii H. Monotypic epithelioid angiomyolipoma of the liver. Histopathology. 2000 May;36(5):451-6. doi: 10.1046/j.1365-2559.2000.00848.x. PMID: 10792487.

5. Martignoni, G.; Pea, M.; Reghellin, D.; Zamboni, G.; Bonetti, F. PEComas: The past, the present and the future.Virchows Arch.2008,452, 119-132. [CrossRef]

6. Dickson MA, Schwartz GK, Antonescu CR. Extrarenal perivascular epithelioid cell tumors (PEComas) respond to mTOR inhibition: clinical and molecular correlates. Int J Cancer. 2013 Apr 1;132(7):17117. doi: 10.1002/ijc.27800. Epub 2012 Sep 21. PMID: 22927055; PMCID: PMC3558545. 
7. Tan, Y .; Xiao, E.H. Hepatic perivascular epithelioid cell tumor (PEComa): Dynamic CT, MRI, ultrasonography, and pathologic features-Analysis of 7 cases and review of the literature.Abdom. Imaging2012,37, 781. [CrossRef] [PubMed]

8. Son, H.-J.; Kang, D.W.; Kim, J.H.; Han, H.Y .; Lee, M.K. Hepatic perivascular epithelioid cell tumor (PEComa): A case report with a review of literatures.Clin. Mol. Hepatol.2017,23, 80-86. [CrossRef]

9. Doyle, L.A.; Argani, P .; Hornick, J.L. PEComa. InWorld Health Organization Classification of Tumours of Soft Tissue and Bone, 5th ed.,IARC Press: Lyon, France, 2020; pp. 312-314.

10. Martignoni, G.; Pea, M.; Reghellin, D.; Zamboni, G.; Bonetti, F. PEComas: The past, the present and the future.Virchows Arch.2008,452, 119-132. [CrossRef]

11. Folpe AL, Mentzel T, Lehr HA. Perivascular epithelioid cell neoplasms of soft tissue and gynecologic origin: a clinicopathologic study of 26 cases and review of the literature. Am J Surg Pathol. 2005 Dec;29(12):1558-75. doi: 10.1097/01.pas.0000173232.22117.37. PMID: 16327428.

12. Hornick JL, Fletcher CD. PEComa: what do we know so far? Histopathology. 2006 Jan;48(1):75-82. doi: 10.1111/j.1365-2559.2005.02316.x. PMID: 16359539.

\section{Figures}
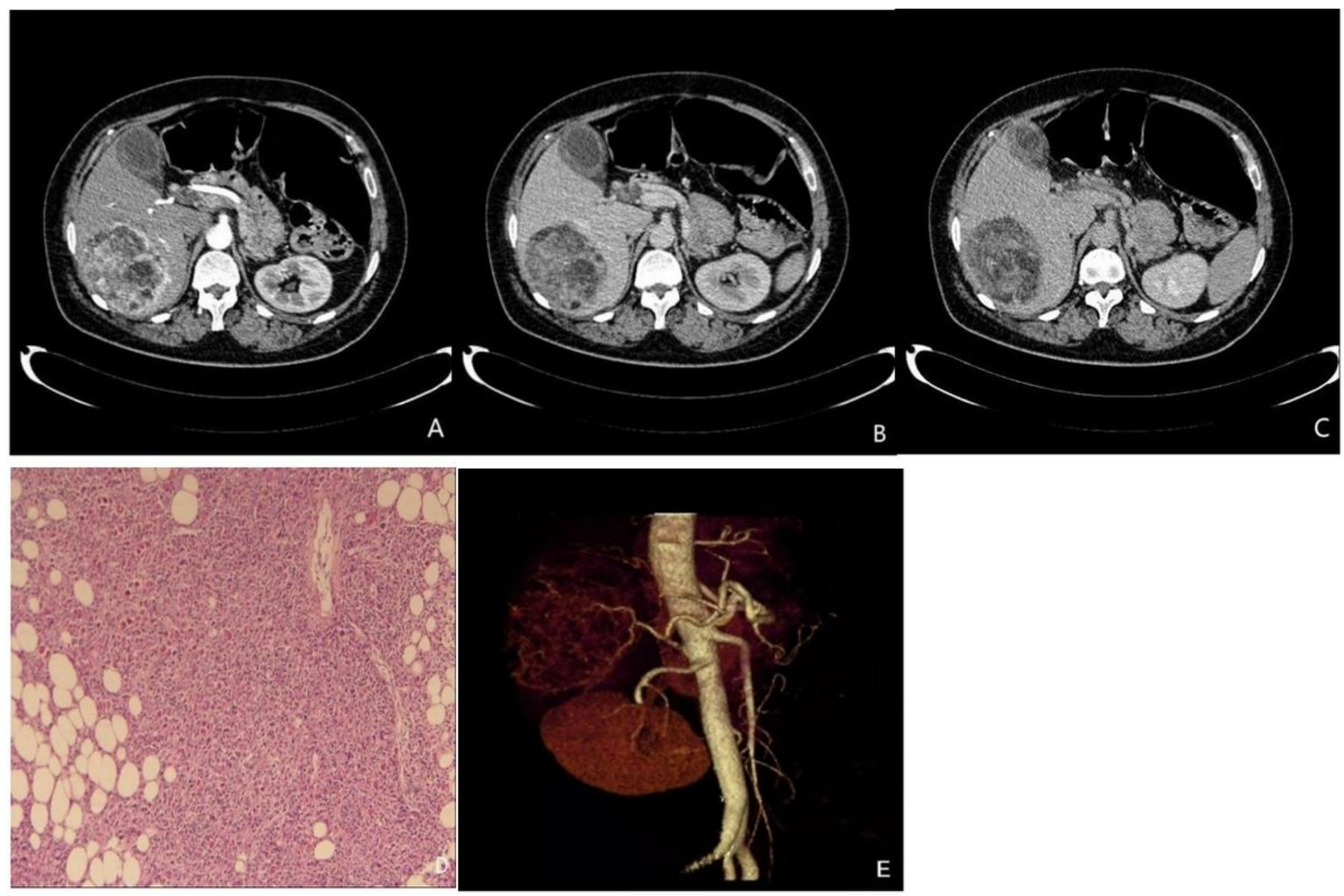


\section{Figure 1}

Axial computed tomography shows an enhancing tumor in the right lobe of the liver which one's adipose tissue was abundant . Patchy enhancement of solid components in arterial phase $(A)$, and there are many small tortuous arteries around and inside the mass. In portal vein phase(B) and delayed phase(C), the mass washed out quickly, and the density was significantly lower than that of the surrounding normal liver parenchyma. Photomicrograph of the tumor, hematoxylin and eosin stain, compose of varying amounts of smooth muscle cells, adipose tissue, and blood vessels. Original magnification, $\times 100$ (D). Reconstructed image(E) showed thick vascular shadow around the tumor and dense vascular network in the tumor.
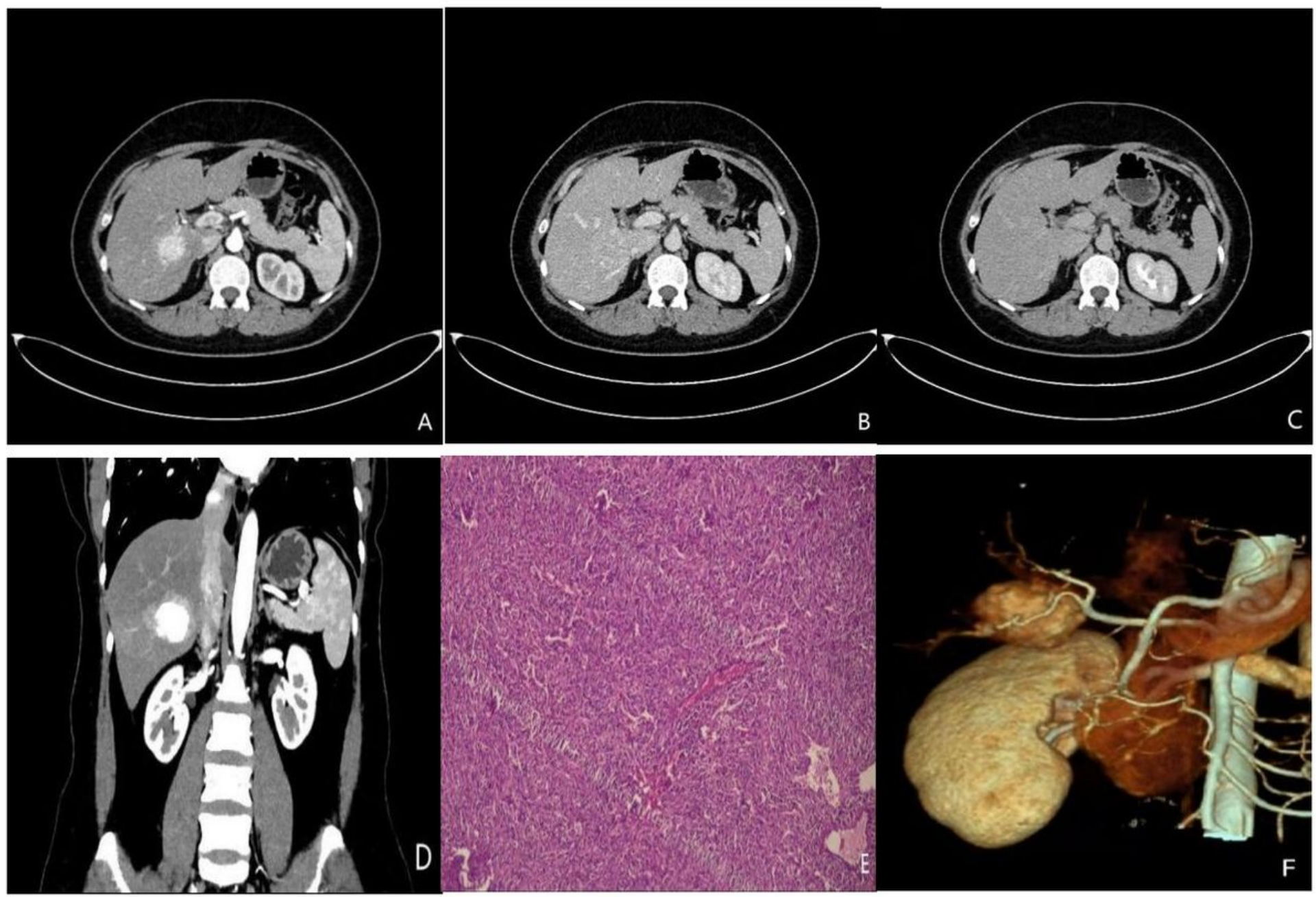

\section{Figure 2}

Axial enhancement CT showed a round isodense mass in the 『segment of the right liver with clear margin. The mass was obviously uniformly enhanced in the arterial phase $(A, D)$. In the portal venous $(B)$ and delayed phase $(\mathrm{C})$, the lesions returned to an isoattenuating stateand decreased enhancement. The nuclear volume of tumor cells and nucleo-plasma ratio increased, pathological nuclear mitosis was visible, and there were more megakaryocytes and singular nuclear cells.Areas of necrosis were also seen, 
and there was no obvious fatty component. Original magnification, $\times 100(D)$. Reconstructed image(F) showeda thick feeding artery. 